Pacific

Journal of

Mathematics

\title{
MAGNETIC RIGIDITY OF HOROCYCLE FLOWS
}

Gabriel P. Paternain

Volume $225 \quad$ No. 2

June 2006 


\title{
MAGNETIC RIGIDITY OF HOROCYCLE FLOWS
}

\author{
GABRiEL P. PATERNAin
}

Let $M$ be a closed oriented surface endowed with a Riemannian metric $g$ and let $\Omega$ be a 2 -form. We show that the magnetic flow of the pair $(g, \Omega)$ has zero asymptotic Maslov index and zero Liouville action if and only if $g$ has constant Gaussian curvature, $\Omega$ is a constant multiple of the area form of $g$ and the magnetic flow is a horocycle flow.

This characterization of horocycle flows implies that if the magnetic flow of a pair $(g, \Omega)$ is $C^{1}$-conjugate to the horocycle flow of a hyperbolic metric $\bar{g}$, there exists a constant $a>0$ such that $a g$ and $\bar{g}$ are isometric and $a^{-1} \Omega$ is, up to a sign, the area form of $g$. It also implies that if a magnetic flow is Mañé-critical and uniquely ergodic it must be the horocycle flow.

As a byproduct we show the existence of closed magnetic geodesics for almost all energy levels in the case of weakly exact magnetic fields on closed manifolds of arbitrary dimension satisfying a certain technical condition.

\section{Introduction}

Let $\Gamma$ be a cocompact lattice of $\operatorname{PSL}(2, \mathbb{R})$. The standard horocycle flow $h$ is given by the right action of the one-parameter subgroup

$$
\left(\begin{array}{ll}
1 & t \\
0 & 1
\end{array}\right)
$$

on $\Gamma \backslash \operatorname{PSL}(2, \mathbb{R})$. The horocycle flow is known to display very peculiar ergodic properties. It preserves the Riemannian volume on $\Gamma \backslash \operatorname{PSL}(2, \mathbb{R})$, is uniquely ergodic [Furstenberg 1973], and mixing of all degrees [Marcus 1978]. It has zero entropy, since

$$
\phi_{t}^{0} \circ h_{s}=h_{s e^{-t}} \circ \phi_{t}^{0}
$$

for all $s, t \in \mathbb{R}$, where $\phi^{0}$ is the geodesic flow given by the one-parameter subgroup

$$
\left(\begin{array}{cc}
e^{t / 2} & 0 \\
0 & e^{-t / 2}
\end{array}\right)
$$

MSC2000: 37D40, 53D25, 37C27.

Keywords: magnetic flow, horocycle flow, Aubry-Mather theory. 
In fact, $h$ parametrizes the strong stable manifold of $\phi^{0}$. The horocycle flow is a very rigid object, as shown in [Marcus 1983; Ratner 1982; 1983]. Recent results on ergodic averages and solutions of cohomological equations for $h$ can be found in [Burger 1990; Flaminio and Forni 2003].

Here we look at horocycle flows as magnetic flows. A matrix $X$ in $\mathfrak{s l}(2, \mathbb{R})$ gives rise to a flow $\phi$ on $\Gamma \backslash \operatorname{PSL}(2, \mathbb{R})$ by setting

$$
\phi_{t}(\Gamma g)=\Gamma g e^{t X} .
$$

The geodesic and horocycle flows are just particular cases of these algebraic flows. Consider the path of matrices in $\mathfrak{s l}(2, \mathbb{R})$ given by

$$
\mathbb{R} \ni \lambda \mapsto X_{\lambda}:=\left(\begin{array}{cc}
1 / 2 & 0 \\
0 & -1 / 2
\end{array}\right)+\lambda\left(\begin{array}{cc}
0 & 1 / 2 \\
-1 / 2 & 0
\end{array}\right)
$$

The flows $\phi^{\lambda}$ on $\Gamma \backslash \operatorname{PSL}(2, \mathbb{R})$ associated with the matrices $X_{\lambda}$ have an interesting interpretation. Since $\operatorname{PSL}(2, \mathbb{R})$ acts by isometries on the hyperbolic plane $\mathbb{H}^{2}$, $M:=\Gamma \backslash \mathbb{T}^{2}$ is a compact hyperbolic surface (provided $\Gamma$ acts without fixed points) and the unit sphere bundle $S M$ of $M$ can be identified with $\Gamma \backslash \operatorname{PSL}(2, \mathbb{R})$. A calculation shows that $\phi^{\lambda}$ is the Hamiltonian flow of the Hamiltonian $H(x, v)=\frac{1}{2}|v|_{x}^{2}$ with respect to the symplectic form on $T M$ given by

$$
-d \alpha+\lambda \pi^{*} \Omega_{a},
$$

where $\Omega_{a}$ is the area form of $M, \pi: T M \rightarrow M$ is the canonical projection and $\alpha$ is the contact 1-form that generates the geodesic flow of $M$. For $\lambda=0, \phi^{0}$ is the geodesic flow and for $\lambda=1, \phi^{1}$ is the flow induced by the one-parameter subgroup with matrix on $\mathfrak{s l}(2, \mathbb{R})$ given by

$$
X_{1}=\left(\begin{array}{rr}
1 / 2 & 1 / 2 \\
-1 / 2 & -1 / 2
\end{array}\right) \text {. }
$$

Since there exists an element $c \in \operatorname{PSL}(2, \mathbb{R})$ such that

$$
c^{-1} X_{1} c=\left(\begin{array}{ll}
0 & 1 \\
0 & 0
\end{array}\right)
$$

the map $f: \Gamma \backslash \operatorname{PSL}(2, \mathbb{R}) \rightarrow \Gamma \backslash \operatorname{PSL}(2, \mathbb{R})$ given by $f(\Gamma g)=\Gamma g c$ conjugates $\phi^{1}$ and $h$; that is, $f \circ \phi_{t}^{1}=h_{t} \circ f$. In fact, any matrix in $\mathfrak{s l}(2, \mathbb{R})$ with determinant zero will give rise to a flow conjugate to $h_{t}$ or $h_{-t}$. (So, up to orientation, there is just one algebraic horocycle flow.) In passing we note that $\operatorname{det} X_{\lambda}=-\frac{1}{4}\left(1-\lambda^{2}\right)$, so for $|\lambda|<1$, the flow $\phi^{\lambda}$ is conjugate to the geodesic flow $\phi^{0}$, up to a constant time scaling by $\sqrt{1-\lambda^{2}}$. Hence the magnetic flows $\phi^{\lambda}$ are just geodesic flows, but with entropy $\sqrt{1-\lambda^{2}}$. This observation is due to V. I. Arnold [1961]. 
In general, if $(M, g)$ is a closed Riemannian manifold and $\Omega$ is a closed 2-form, the Hamiltonian flow $\phi$ of $H(x, v)=\frac{1}{2}|v|_{x}^{2}$ with respect to the symplectic form on $T M$ given by

$$
\omega:=-d \alpha+\pi^{*} \Omega,
$$

is called the magnetic flow of the pair $(g, \Omega)$ because it models the motion of a particle under the influence of the magnetic field $\Omega$. The projection of the orbits of $\phi$ to $M$ are called magnetic geodesics. The discussion above shows that $h$ appears as the magnetic flow of a hyperbolic surface with $\Omega$ equal to the area form of the surface.

Since the horocycle flow has no closed orbits, this already gives an example of a Hamiltonian system with an energy level (the unit sphere bundle $S M$ ) without closed orbits. This example has been much used, most notably by V. Ginzburg [1995; 1996; 1997] to give smooth counterexamples to the Hamiltonian Seifert conjecture in $\mathbb{R}^{2 n}$, where $n \geq 3$. (A $C^{2}$-counterexample is now available in $\mathbb{R}^{4}$; see [Ginzburg and Gürel 2003].)

How often does the horocycle flow appear as a magnetic flow? To answer this we first prove a characterization of horocycle flows within the set of magnetic flows.

Magnetic flows on surfaces leave invariant the volume form $\alpha \wedge d \alpha$. The associated Borel probability measure is called the Liouville measure $\mu_{\ell}$ of $S M$. We shall assume from now on that $M$ has genus $\geq 2$. Then $\pi^{*}: H^{2}(M, \mathbb{R}) \rightarrow H^{2}(S M, \mathbb{R})$ is the zero map and thus if $\Omega$ is any 2 -form, $\pi^{*} \Omega$ is exact on $S M$. It follows that $\omega$ restricted to $S M$ is exact and we let $\Theta$ be any primitive. We define the action of the Liouville measure as

$$
\mathfrak{a}\left(\mu_{\ell}\right):=\int \Theta(X) d \mu_{\ell},
$$

where $X$ is the vector field on $S M$ that generates the magnetic flow. The action does not depend on the primitive, since the asymptotic cycle of $\mu_{\ell}$ is zero (see Section 2 and [Contreras et al. 2003]). That is, for any closed 1-form $\varphi$ on $S M$ we have

$$
\int \varphi(X) d \mu_{\ell}=0
$$

It is simple to check that $\mathfrak{a}\left(\mu_{\ell}\right)=0$ for the horocycle flow, the flow defined on a hyperbolic surface $M$ when $\Omega$ is the area form. In fact, in this case, there is a primitive $\Theta$ with $\Theta(X) \equiv 0$. It is also easy to check that there are no conjugate points [Contreras and Iturriaga 1999, Example A.1]. Equivalently, using the results in [Contreras et al. 2003], we can say that the asymptotic Maslov index $\mathfrak{m}\left(\mu_{\ell}\right)$ of the Liouville measure is zero (see Section 2). (We remark that the only magnetic flows without conjugate points on the 2-torus are geodesic flows of flat metrics; see [Bialy 2000]). 
We first show that these two symplectic-ergodic quantities characterize horocycle flows.

Proposition. Let $M$ be a closed oriented surface of genus greater than or equal to two endowed with a Riemannian metric $g$ and let $\Omega$ be a 2-form. The magnetic flow of the pair $(g, \Omega)$ has $\mathfrak{a}\left(\mu_{\ell}\right)=\mathfrak{m}\left(\mu_{\ell}\right)=0$ if and only if $g$ has constant Gaussian curvature, $\Omega$ is a constant multiple of the area form of $g$ and the magnetic flow is a horocycle flow.

The Proposition has the following consequence:

Theorem A. Let $M$ be a closed oriented surface endowed with a Riemannian metric $g$ and let $\Omega$ be a 2-form. If the magnetic flow of the pair $(g, \Omega)$ is $C^{1}$-conjugate to the horocycle flow of a hyperbolic metric $\bar{g}$, there exists a constant a $>0$, such that $a g$ and $\bar{g}$ are isometric and $a^{-1} \Omega$ is, up to a sign, the area form of $g$.

It is not possible to conclude that $g$ has curvature -1 (i.e. $a=1$ ). This is simply because the magnetic flow of a pair $(g, \Omega)$ with $g$ of constant negative curvature $-k, \Omega=\lambda \Omega_{a}(\lambda>0)$ and $\lambda^{2}=k$ is smoothly conjugate to the horocycle flow of a hyperbolic surface. To see this observe that an easy scaling argument shows that the magnetic flow of a such pair is, up to a constant time change, conjugate to the horocycle flow of a hyperbolic surface. But by (1), $h_{t}$ is conjugate to $h_{\tau t}$ for any positive real number $\tau$. This shows that the area $A$ of a surface is not preserved under $C^{1}$-conjugacies of magnetic flows. C. Croke and B. Kleiner [1994] have shown that the volume of a Riemannian manifold is preserved under $C^{1}$ conjugacies of geodesic flows. In the case of transitive magnetic flows, we show that $C^{1}$-conjugacies preserve $\mathfrak{a}\left(\mu_{\ell}\right) / A$ (Lemma 4.1).

We now describe a second and more involved application of the Proposition. Let $\widetilde{\Omega}$ be the lift of $\Omega$ to the universal cover $\widetilde{M} \cong \mathbb{R}^{2}$ of $M$. Since $\widetilde{\Omega}$ is an exact form, there exists a smooth 1 -form $\theta$ such that $\widetilde{\Omega}=d \theta$. Consider the Lagrangian on $\widetilde{M}$ given by

$$
L(x, v)=\frac{1}{2}|v|_{x}^{2}-\theta_{x}(v) .
$$

It is well known that the extremals of $L$, which are the solutions of the EulerLagrange equations

$$
\frac{d}{d t} \frac{\partial L}{\partial v}(x, v)=\frac{\partial L}{\partial x}(x, v),
$$

coincide with the lift to $\tilde{M}$ of the magnetic geodesics. The action of the Lagrangian $L$ on an absolutely continuous curve $\gamma:[a, b] \rightarrow \widetilde{M}$ is defined by

$$
A_{L}(\gamma)=\int_{a}^{b} L(\gamma(t), \dot{\gamma}(t)) d t
$$


The Mañé critical value of the pair $(g, \Omega)$ is defined as

$c=c(g, \Omega):=\inf \left\{k \in \mathbb{R}: A_{L+k}(\gamma) \geq 0\right.$ for any absolutely continuous

closed curve $\gamma$ defined on any closed interval $[0, T]\}$.

Like any Lagrangian flow, the magnetic flow for $T \tilde{M}$ can be viewed as the Hamiltonian flow defined by the canonical symplectic form on $T^{*} \widetilde{M}$ and a suitable Hamiltonian function $H: T^{*} \widetilde{M} \rightarrow \mathbb{R}$; in this case,

$$
H(x, p)=\frac{1}{2}\left|p+\theta_{x}\right|^{2} .
$$

The Legendre transform $\mathscr{L}: T \widetilde{M} \rightarrow T^{*} \tilde{M}$, defined by

$$
\mathscr{L}(x, v)=\frac{\partial L}{\partial v}(x, v),
$$

carries orbits of the Lagrangian flow for $L$ to orbits of the Hamiltonian flow defined by $H$ and the canonical symplectic form. The critical value can also be defined in Hamiltonian terms [Burns and Paternain 2002] as:

$$
c(g, \Omega)=\inf _{u \in C^{\infty}(\tilde{M}, \mathbb{R})} \sup _{x \in \tilde{M}} H\left(x, d_{x} u\right)=\inf _{u \in C^{\infty}(\tilde{M}, \mathbb{R})} \sup _{x \in \tilde{M}} \frac{1}{2}\left|d_{x} u+\theta_{x}\right|^{2} .
$$

As $u$ ranges over $C^{\infty}(\tilde{M}, \mathbb{R})$ the form $\theta+d u$ ranges over all primitives of $\widetilde{\Omega}$, because any two primitives differ by a closed 1 -form, which must be exact since $\widetilde{M}$ is simply connected. Since for a surface of genus at least 2 there are always bounded primitives, we have $c(g, \Omega)<\infty$. (On the upper-half plane with the hyperbolic metric, the primitive $-d x / y$ of the area form $d x \wedge d y / y^{2}$ is bounded.)

We will say that a magnetic flow is Mañé-critical if $c(g, \Omega)=\frac{1}{2}$. Magnetic flows which are supercritical, i.e. $\frac{1}{2}>c(g, \Omega)$ always have positive topological entropy [Burns and Paternain 2002, Proposition 5.4]. Hence such flows exhibit a horseshoe and exponential growth rate of hyperbolic closed magnetic geodesics. In fact, we will show that, in any dimension, if $\frac{1}{2}>c(g, \Omega)$, then a nontrivial homotopy class contains a closed magnetic geodesic provided that the centralizer of some element in the class is an amenable subgroup (Theorem 5.5). For subcritical magnetic flows - those for which $\frac{1}{2}<c(g, \Omega)$ - one hopes to prove that there are always closed contractible magnetic geodesics, although nothing of this kind has been proved in general. When $\Omega$ itself is exact, the main result in [Contreras et al. 2004] says that there always exists a closed magnetic geodesic.

What happens for magnetic flows which are Mañé-critical with $\Omega$ nonexact? It is easy to check that the horocycle flow is Mañé-critical; see [Contreras 2001, Example 6.2]. Is it the only magnetic flow which is Mañé-critical and uniquely ergodic? Aubry-Mather theory combined with the Proposition gives the answer: 
Theorem B. Let $M$ be a closed oriented surface endowed with a Riemannian metric $g$ and let $\Omega$ be a 2-form. Suppose the magnetic flow of the pair $(g, \Omega)$ is Mañe-critical and uniquely ergodic. Then $g$ has constant Gaussian curvature, $\Omega$ is a constant multiple of the area form of $g$ and the magnetic flow is a horocycle flow.

Our results on the existence of closed magnetic geodesics in nontrivial free homotopy classes for supercritical magnetic flows, combined with recent results of G. Contreras [2003] and O. Osuna [2005], imply the following statement on the almost existence of closed magnetic geodesics for weakly exact magnetic flows. Recall that $\Omega$ is weakly exact if its lift to the universal covering $\widetilde{M}$ of $M$ is exact.

Theorem C. Let $M$ be an arbitrary closed manifold endowed with a Riemannian metric $g$ and let $\Omega$ be a weakly exact 2-form whose lift to $\widetilde{M}$ has a bounded primitive. If $\pi_{1}(M)$ contains a nontrivial element with an amenable centralizer, then almost every energy level contains a closed magnetic geodesic.

Recall that a discrete group $\Gamma$ is said to be amenable if the space of bounded functions $\Gamma \rightarrow \mathbb{R}$ has a left (or right) invariant mean [Pier 1984]. Examples are finite groups, abelian groups and finite extensions of solvable groups. If a group contains a free subgroup on two generators, then it is nonamenable. I do not know of any example of a finitely presented group for which the centralizer of every element is nonamenable. Contreras [2003] proved Theorem $C$ when $\Omega$ is exact without any assumption on $\pi_{1}(M)$ and V. Ginzburg and E. Kerman [1999] proved the theorem when $M$ is a torus. Also, by virtue of Preissman's theorem, Theorem $\mathrm{C}$ applies to all closed manifolds that admit a metric of negative curvature (in this case there are bounded primitives; see [Gromov 1991]).

There are several results establishing the existence of closed contractible magnetic geodesics for almost every low energy level; see [Schlenk 2006; Macarini 2003] and references therein. The methods of symplectic topology have proved to be effective in this respect. For high energy levels, at least when $g$ is generic, one can also obtain existence of closed magnetic geodesics in free homotopy classes by observing that magnetic flows approach geodesic flows as energy increases. Very little is known about how to bridge the gap. The exceptions seem to be Theorem $\mathrm{C}$ and the main result in [Contreras et al. 2004] alluded above, which are both based on Aubry-Mather theory.

\section{Geometric preliminaries}

Let $M$ be a closed oriented surface, $S M$ the unit sphere bundle and $\pi: S M \rightarrow M$ the canonical projection. The latter is in fact a principal $S^{1}$-fibration and we let $V$ be the infinitesimal generator of the action of $S^{1}$. 
Given a unit vector $v \in T_{x} M$, we will denote by $i v$ the unique unit vector orthogonal to $v$ such that $\{v, i v\}$ is an oriented basis of $T_{x} M$. There are two basic 1 -forms $\alpha$ and $\beta$ on $S M$, defined by the formulas

$$
\alpha_{(x, v)}(\xi):=\left\langle d_{(x, v)} \pi(\xi), v\right\rangle, \quad \beta_{(x, v)}(\xi):=\left\langle d_{(x, v)} \pi(\xi), i v\right\rangle .
$$

The form $\alpha$ is precisely the contact form that we mentioned in the Introduction. The vector field $X_{0}$ uniquely determined by the equations $\alpha\left(X_{0}\right) \equiv 1$ and $i_{X_{0}} d \alpha \equiv 0$ generates the geodesic flow $\phi^{0}$ of $M$.

A basic theorem in two-dimensional Riemannian geometry asserts that there exists a unique 1-form $\psi$ on $S M$ (the connection form) such that $\psi(V) \equiv 1$ and

$$
d \alpha=\psi \wedge \beta, \quad d \beta=-\psi \wedge \alpha, \quad d \psi=-(K \circ \pi) \alpha \wedge \beta,
$$

where $K$ is the Gaussian curvature of $M$. In fact, the form $\psi$ is given by

$$
\psi_{(x, v)}(\xi)=\left\langle\frac{D Z}{d t}(0), i v\right\rangle
$$

where $Z:(-\varepsilon, \epsilon) \rightarrow S M$ is any curve with $Z(0)=(x, v)$ and $\dot{Z}(0)=\xi$, and $D Z / d t$ is the covariant derivative of $Z$ along the curve $\pi \circ Z$.

It is easy to check that $\alpha \wedge \beta=\pi^{*} \Omega_{a}$, where $\Omega_{a}$ is the area form. Hence

$$
d \psi=-\pi^{*}\left(K \Omega_{a}\right) .
$$

In the case of a hyperbolic surface, the vertical vector field $V$ corresponds to the matrix in $\mathfrak{s l}(2, \mathbb{R})$ given by

$$
\left(\begin{array}{cc}
0 & 1 / 2 \\
-1 / 2 & 0
\end{array}\right)
$$

Asymptotic cycles. Given a $\phi$-invariant Borel probability measure $\mu$, the asymptotic cycle of $\mu$ is the real 1-homology class $\mathscr{S}(\mu)$ defined by the equality

$$
\langle[\varphi], \mathscr{S}(\mu)\rangle=\int_{S M} \varphi(X) d \mu
$$

for any closed 1-form $\varphi$. (Recall that $\phi$ is the magnetic flow of the pair $(g, \Omega)$ with associated vector field $X$.) We check that $\mathscr{Y}\left(\mu_{\ell}\right)=0$. We will prove a slightly more general result needed later. Let $\Lambda_{0}^{*}$ be the space of continuous forms $\lambda$ whose exterior derivatives, weakly defined by Stokes' theorem $\left(\int_{\sigma} d \lambda=\int_{\partial \sigma} \lambda\right.$ for every smooth chain $\sigma$ ) are also continuous differential forms. The space $\Lambda_{0}^{*}$ is closed under exterior differentiation, wedge products and pull back by $C^{1}$ maps.

Let $\varphi$ be a continuous 1-form that is closed in the sense that its integral over the boundary of any 2-chain is zero: $\varphi \in \Lambda_{0}^{1}$ and $d \varphi=0$. We claim that

$$
\int_{S M} \varphi(X) d \mu_{\ell}=0 .
$$


Note that we always have $\alpha \wedge \pi^{*} \Omega=0$ as it easily follows from evaluating the 3 -form on any basis that contains $V$. Thus $\alpha \wedge(-d \alpha)=\alpha \wedge \omega\left(\omega=-d \alpha+\pi^{*} \Omega\right)$ and it suffices to show that

Observe that

$$
\int_{S M} \varphi(X) \alpha \wedge \omega=0
$$

$$
\varphi(X) \alpha \wedge \omega=\varphi \wedge i_{X}(\alpha \wedge \omega)=\varphi \wedge \omega,
$$

since $i_{X} \omega=0$. But since $\omega$ is exact, if we let $\Theta$ be a primitive, we have $d(\varphi \wedge \Theta)=$ $\varphi \wedge \omega$ and the claim follows from our definition of exterior differentiation and the fact that $M$ is a closed surface.

Asymptotic Maslov index. Let $\Lambda(S M)$ be the set of Lagrangian subspaces of the restriction $\left.T\right|_{S M} T M$. Given any subspace $E \in \Lambda(S M)$ at $(x, v)$ and any $T>0$, we can consider the curve of Lagrangian subspaces $[0, T] \ni t \mapsto d \phi_{t}(E)$. Let $n(x, v, E, T)$ be the intersection number of this curve with the Maslov cycle of $\Lambda(S M)$. It was shown in [Contreras et al. 2003] that if $\mu$ is $\phi$-invariant, the limit

$$
\mathfrak{m}(x, v):=\lim _{T \rightarrow \infty} \frac{1}{T} n(x, v, E, T)
$$

exists for $\mu$-almost every $(x, v)$, is independent of $E$, and $(x, v) \mapsto \mathfrak{m}(x, v)$ is integrable. The asymptotic Maslov index of $\mu$ is

$$
\mathfrak{m}(\mu):=\int_{S M} \mathfrak{m}(x, v) d \mu(x, v) .
$$

Green subbundles. Given $(x, v) \in T M$ we define the vertical subspace at $(x, v)$ as $\mathscr{V}(x, v):=\operatorname{ker} d_{(x, v)} \pi$, where $\pi: T M \rightarrow M$ is the canonical projection. Note that $\mathscr{V}(x, v) \cap T_{(x, v)} S M$ is spanned by the value of the vector field $V$ at $(x, v)$. We say that the orbit of $(x, v) \in S M$ does not have conjugate points if, for all $t \neq 0$,

$$
d_{(x, v)} \phi_{t}(\mathscr{V}(x, v)) \cap \mathscr{V}\left(\phi_{t}(x, v)\right)=\{0\} .
$$

Since magnetic flows are optical, the main result of [Contreras et al. 2003] says that $S M$ has no conjugate points (meaning that no orbit a point $(x, v) \in M$ has conjugate points) if and only if $\mathfrak{m}\left(\mu_{\ell}\right)=0$.

If $S M$ has no conjugate points, one can construct the Green subbundles [Contreras and Iturriaga 1999, Proposition A] given by

$$
E(x, v):=\lim _{t \rightarrow+\infty} d \phi_{-t}\left(\mathscr{V}\left(\phi_{t}(x, v)\right)\right), \quad F(x, v):=\lim _{t \rightarrow+\infty} d \phi_{t}\left(\mathscr{V}\left(\phi_{-t}(x, v)\right)\right) .
$$

These subbundles are Lagrangian, they never intersect the vertical subspace and, crucially for us, they are contained in $T(S M)$. Moreover, they vary measurably with $(x, v)$ and they contain the vector field $X$. 


\section{Proof of the Proposition}

The proof will be based on integrating an appropriate Riccati equation along a solution arising from the Green bundles. This is a well known method, first used by E. Hopf [1948] and subsequently extended to higher dimensions by L. W. Green [1958]. The method is still paying dividends; see [Bialy 2000; Gouda 1998].

Let $\Omega$ be an arbitrary smooth 2-form. We write $\Omega=f \Omega_{a}$, where $f: M \rightarrow \mathbb{R}$ is a smooth function and $\Omega_{a}$ is the area form of $g$.

Since $H^{2}(M, \mathbb{R})=\mathbb{R}$, there exist a constant $c$ and a smooth 1 -form $\varrho$ such that

$$
\Omega=c K \Omega_{a}+d \varrho
$$

and $c=0$ if and only if $\Omega$ is exact. Using (2) we have

$$
\omega:=-d \alpha+\pi^{*} \Omega=d\left(-\alpha-c \psi+\pi^{*} \varrho\right) .
$$

The vector field $X$ that generates the magnetic flow $\phi$ is given by $X=X_{0}+f V$ since it satisfies the equation $d H=i_{X} \omega$. Since $X_{0}$ and $V$ preserve the volume form $\alpha \wedge d \alpha$, so does $X=X_{0}+f V$, and thus $\phi$ preserves the normalized Liouville measure $\mu_{\ell}$ of $S M$.

If we evaluate the primitive $-\alpha-c \psi+\pi^{*} \varrho$ of the symplectic form $\omega$ on $X$ we obtain:

$$
\left(-\alpha-c \psi+\pi^{*} \varrho\right)(X)(x, v)=-1-c f(x)+\varrho_{x}(v) .
$$

Let $A$ be the area of $g$. Then $\mathfrak{a}\left(\mu_{\ell}\right)$ is given by:

$$
\mathfrak{a}\left(\mu_{\ell}\right)=-1-\frac{c}{A} \int_{M} f(x) d x
$$

since $\mu_{\ell}$ is invariant under the flip $v \mapsto-v$. By the definition of $c$ and $f$ and the Gauss-Bonnet theorem we have

$$
\int_{M} f(x) d x=c \int_{M} K(x) d x=2 \pi \chi c,
$$

and hence

$$
\mathfrak{a}\left(\mu_{\ell}\right)=-1-\frac{1}{2 \pi \chi A}\left(\int_{M} f(x) d x\right)^{2} .
$$

Given $(x, v) \in S M$ and $\xi \in T_{(x, v)} T M$, let

$$
J_{\xi}(t)=d_{(x, v)}\left(\pi \circ \phi_{t}\right)(\xi) .
$$

We call $J_{\xi}$ a magnetic Jacobi field with initial condition $\xi$. It was shown in [Paternain and Paternain 1996] that $J_{\xi}$ satisfies the Jacobi equation

$$
\ddot{J}_{\xi}+R\left(\dot{\gamma}, J_{\xi}\right) \dot{\gamma}-\left(Y\left(\dot{J}_{\xi}\right)+\left(\nabla_{J_{\xi}} Y\right)(\dot{\gamma})\right)=0,
$$


where $\gamma(t)=\pi \circ \phi_{t}(x, v), R$ is the curvature tensor of $g$ and $Y$ is determined by the equality $\Omega_{x}(u, v)=\left\langle Y_{x}(u), v\right\rangle$ for all $u, v \in T_{x} M$ and all $x \in M$.

We express $J_{\xi}$ as

$$
J_{\xi}(t)=x(t) \dot{\gamma}(t)+y(t) i \dot{\gamma}(t),
$$

and suppose in addition that $\xi \in T_{(x, v)} S M$, which implies that

$$
g_{\gamma}\left(\dot{J}_{\xi}, \dot{\gamma}\right)=0 \text {. }
$$

A straightforward computation using this equality and (5) shows that $x$ and $y$ must satisfy the scalar equations

$$
\dot{x}=f(\gamma) y, \quad \ddot{y}+\left(K(\gamma)-\langle\nabla f(\gamma), i \dot{\gamma}\rangle+f^{2}(\gamma)\right) y=0 .
$$

The no-conjugate points condition is equivalent to saying that any nontrivial magnetic Jacobi field which vanishes at $t=0$ is never zero again.

Consider one of the Green subbundles, say $E$. Since $E$ does not intersect the vertical subspace $\mathscr{V}(x, v)$ for any $(x, v) \in S M$, there exists a linear map $S(x, v)$ : $T_{x} M \rightarrow T_{x} M$ such that $E$ can be identified with the graph of $S$. Let $u(x, v)$ be the trace of $S(x, v)$. An easy calculation using (6) shows that $u$ along $\phi$ satisfies the Riccati equation

$$
\dot{u}+u^{2}+K(\gamma)-\langle\nabla f(\gamma), i \dot{\gamma}\rangle+f^{2}(\gamma)=0 .
$$

We can now integrate equation (7) with respect to $t \in[0,1]$ and then with respect to $\mu_{\ell}$ (using the $\phi$-invariance of $\mu_{\ell}$ ) to conclude that

$$
\int_{S M} u^{2} d \mu_{\ell}+\int_{S M}\left(K(x)-\langle\nabla f(x), i v\rangle+f^{2}(x)\right) \mu_{\ell}=0 .
$$

Since $\mu_{\ell}$ is invariant under the flip $v \mapsto-v$, we have

and thus

$$
\int_{S M}\langle\nabla f(x), i v\rangle d \mu_{\ell}=0
$$

$$
\int_{S M} u^{2} d \mu_{\ell}+\int_{S M}\left(K(x)+f^{2}(x)\right) \mu_{\ell}=0 .
$$

The last equality implies that

$$
\int_{S M}\left(K(x)+f^{2}(x)\right) \mu_{\ell}=\frac{2 \pi \chi}{A}+\frac{1}{A} \int_{M} f^{2}(x) d x \leq 0,
$$

with equality if and only if $u$ is zero for almost every $(x, v) \in S M$. But if we now assume that the action $\mathfrak{a}\left(\mu_{\ell}\right)$ vanishes, equation (4) and the Cauchy-Schwarz inequality tell us that

$$
-2 \pi \chi A=\left(\int_{M} f(x) d x\right)^{2} \leq A \int_{M} f^{2}(x) d x .
$$


Combining the last inequality with (8) we see that $f$ must be constant and $u$ is zero for almost every $(x, v) \in S M$. If we now use this information in the Riccati equation (7) we conclude that $K$ must be constant and $K+f^{2}=0$. The last equality ensures that the magnetic flow is a horocycle flow, thus concluding the proof of the Proposition.

\section{Proof of Theorem A}

Lemma 4.1. Let $M_{i}$, for $i=1,2$, be closed oriented surfaces with magnetic flows $\phi^{i}$ determined by pairs $\left(g_{i}, \Omega_{i}\right), i=1,2$. Suppose $\phi^{1}$ is $C^{1}$-conjugate to $\phi^{2}$ and one of them is transitive. Then

$$
A_{2} \mathfrak{a}\left(\mu_{\ell}^{1}\right)=A_{1} \mathfrak{a}\left(\mu_{\ell}^{2}\right),
$$

where $A_{i}$ is the area of $g_{i}$.

Proof. Let $f: S M_{1} \rightarrow S M_{2}$ be the $C^{1}$-conjugacy and $\omega_{i}$ the corresponding symplectic forms restricted to $S M_{i}$. Recall that $\alpha \wedge(-d \alpha)=\alpha \wedge \omega$. Since magnetic flows preserve $\alpha \wedge d \alpha$, the volume form $f^{*}\left(\alpha_{2} \wedge \omega_{2}\right)$ is invariant under $\phi^{1}$. Since we are assuming that the magnetic flows are transitive, there exists a (nonzero) constant $\kappa$ such that

$$
f^{*}\left(\alpha_{2} \wedge \omega_{2}\right)=\kappa \alpha_{1} \wedge \omega_{1} .
$$

Note that $d f$ maps $X_{1}$ to $X_{2}$, that $\alpha_{i}\left(X_{i}\right)=1$ and that $i_{X_{i}} \omega_{i}=0$. Hence contracting with $X_{1}$ in the last equation gives

$$
f^{*} \omega_{2}=\kappa \omega_{1} .
$$

Let $\Theta_{i}$ be a primitive of $\omega_{i}$. Then $\varphi:=f^{*} \Theta_{2}-\kappa \Theta_{1}$ is a continuous 1-form, which is closed in the sense that its integral over the boundary of every 2-chain is zero. By (9), $f_{*} \mu_{\ell}^{1}=\mu_{\ell}^{2}$, thus

$$
\int_{S M_{1}} \varphi\left(X_{1}\right) d \mu_{\ell}^{1}=\int_{S M_{2}} \Theta_{2}\left(X_{2}\right) d \mu_{\ell}^{2}-\kappa \int_{S M_{1}} \Theta_{1}\left(X_{1}\right) d \mu_{\ell}^{1}=\mathfrak{a}\left(\mu_{\ell}^{2}\right)-\kappa \mathfrak{a}\left(\mu_{\ell}^{1}\right) .
$$

But, since the asymptotic cycle of $\mu_{\ell}$ is zero (page 307), the left-hand side vanishes. Equality (9) implies that $\kappa=A_{2} / A_{1}$ and the lemma follows.

Lemma 4.2. Let $M_{i}$, for $i=1,2$, be closed oriented surfaces with magnetic flows $\phi^{i}$ determined by pairs $\left(g_{i}, \Omega_{i}\right)$. Suppose $\phi^{1}$ is $C^{1}$-conjugate to $\phi^{2}$ and let $f$ : $S M_{1} \rightarrow S M_{2}$ be the conjugacy. Then $\mathfrak{m}\left(\mu_{\ell}^{1}\right)=\mathfrak{m}\left(f_{*} \mu_{\ell}^{1}\right)$. In particular, $\phi^{1}$ has conjugate points if and only if $\phi^{2}$ does.

Proof. Let $W_{1}(x, v)$ be the subspace of $T_{(x, v)} S M_{1}$ spanned by the magnetic vector field $X_{1}$ and the vertical vector field $V$. Since $W_{1}$ contains the magnetic vector field and it is 2-dimensional it must be a Lagrangian subbundle. Since $d f$ maps 
$X_{1}$ to $X_{2}$, it also maps Lagrangian subspaces contained in $T\left(S M_{1}\right)$ to Lagrangian subspaces contained in $T\left(S M_{2}\right)$. In particular, the subbundle $W_{2}:=d f\left(W_{1}\right)$ must be a Lagrangian subbundle contained in $T\left(S_{2}\right)$.

We now invoke [Contreras et al. 2003, Corollary 3.2], to the effect that the asymptotic Maslov index of a measure with zero asymptotic cycle does not depend on the continuous Lagrangian section that is used to compute it. Thus we can compute $\mathfrak{m}\left(\mu_{\ell}^{1}\right)$ using $W_{1}$ and $\mathfrak{m}\left(f_{*} \mu_{\ell}^{1}\right)$ using $W_{2}$ to readily obtain the equality claimed in the lemma. To see that $\phi^{1}$ has conjugate points if and only if $\phi^{2}$ does we use [Contreras et al. 2003, Theorem 4.4], which says that the asymptotic Maslov index of an invariant probability measure (with zero asymptotic cycle) is positive if and only if there are conjugate points in its support.

We now prove Theorem A. We know that the horocycle flow of a closed hyperbolic surface has $\mathfrak{a}\left(\mu_{\ell}\right)=\mathfrak{m}\left(\mu_{\ell}\right)=0$. Since the horocycle flow is transitive, by Lemmas 4.1 and 4.2 the magnetic flow of $(g, \Omega)$ also has $\mathfrak{a}\left(\mu_{\ell}\right)=\mathfrak{m}\left(\mu_{\ell}\right)=0$. The Proposition tells us that $g$ has constant curvature $k$ and $\Omega=\lambda \Omega_{a}$ with $k+\lambda^{2}=0$. If we let $a:=-k$, then $a g$ has curvature -1 and we have the situation of two closed hyperbolic surfaces with $C^{1}$-conjugate horocycle flows. From [Marcus 1983] or [Ratner 1982] we know that $a g$ and $\bar{g}$ must in fact be isometric, as desired.

\section{Closed orbits in nontrivial free homotopy classes}

We consider magnetic flows defined on an arbitrary closed connected manifold $M$. Let $g$ be a Riemannian metric and let $\Omega$ be a closed 2-form. We will assume that $\Omega$ is weakly exact, that is, the lift of $\Omega$ to $\widetilde{M}$, the universal covering of $M$, is exact. Let $\theta$ be a primitive and let $c=c(g, \Omega)$ be Mañé's critical value, defined as in the Introduction. Recall that $c$ is finite if and only if there is a bounded primitive. If $\theta$ is a bounded primitive, then our Lagrangian $L$ satisfies all the hypotheses of AubryMather theory for noncompact manifolds as described for example in [Contreras 2001; Fathi $\geq 2007$; Fathi and Maderna $\geq 2007$ ]. Recall that the energy in this case is simply the real valued function on $T \tilde{M}$ given by $(x, v) \mapsto \frac{1}{2}|v|_{x}^{2}$.

We consider $\pi_{1}(M)$ acting on $\tilde{M}$ by covering transformations and we let $\Pi$ : $\tilde{M} \rightarrow M$ be the covering projection. Given a nontrivial element $\varphi \in \pi_{1}(M)$, let $Z_{\varphi}:=\left\{\rho \in \pi_{1}(M): \rho^{-1} \varphi \rho=\varphi\right\}$ be the centralizer of $\varphi$.

Theorem 5.1. Let $k>c$ be given. Suppose that there exists a primitive $\theta$ which is $Z_{\varphi}$-invariant and for which

$$
\sup _{x \in M} \frac{1}{2}\left|\theta_{x}\right|^{2} \leq k-\varepsilon
$$

for some $\varepsilon>0$. Then the nontrivial free homotopy class determined by $\varphi$ contains a closed magnetic geodesic with energy $k$. 
Proof. Let $\theta$ be a $Z_{\varphi}$-invariant primitive with

$$
\sup _{x \in M} \frac{1}{2}\left|\theta_{x}\right|^{2} \leq k-\varepsilon
$$

for some $\varepsilon>0$. If we consider the Lagrangian on $\widetilde{M}$ given by

$$
L(x, v)=\frac{1}{2}|v|_{x}^{2}-\theta_{x}(v)
$$

then

$$
L(x, v)+k \geq \varepsilon>0
$$

for all $(x, v) \in T M$. Consider the Mañé action potential, which is given by

$$
\Phi_{k}(x, y)=\inf _{T>0} \Phi_{k}(x, y ; T),
$$

where

$$
\Phi_{k}(x, y ; T):=\inf _{\gamma} A_{L+k}(\gamma)
$$

with $\gamma$ ranging over all absolutely continuous curves defined on $[0, T]$ and connecting $x$ to $y$. The potential $\Phi_{k}$ is a Lipschitz function and satisfies the triangle inequality:

$$
\Phi_{k}(x, y) \leq \Phi_{k}(x, z)+\Phi_{k}(z, y) .
$$

Note that the action potential is $Z_{\varphi}$-invariant, since $\theta$ is $Z_{\varphi}$-invariant.

Let $\psi \in \pi_{1}(M)$ be an arbitrary covering transformation. Since $\psi^{*} \theta-\theta$ is closed, there exists a smooth function $f_{\psi}: \widetilde{M} \rightarrow \mathbb{R}$ such that $\psi^{*} \theta-\theta=d f_{\psi}$. The function $f_{\psi}$ is uniquely defined up to addition of a constant, so from now on we shall assume that $f_{\psi}$ is the unique function for which $f_{\psi}\left(x_{0}\right)=0$ where $x_{0}$ is some fixed point in $\widetilde{M}$. Note that from the definition of $\Phi_{k}$ we have:

$$
\Phi_{k}(\psi x, \psi y)=\Phi_{k}(x, y)+f_{\psi}(y)-f_{\psi}(x)
$$

for all $x, y \in \tilde{M}$ and all $\psi \in \pi_{1}(M)$.

Lemma 5.2. If $\psi_{1}^{-1} \varphi \psi_{1}=\psi_{2}^{-1} \varphi \psi_{2}$, then $f_{\psi_{1}}=f_{\psi_{2}}$.

Proof. Clearly $\tau:=\psi_{1} \psi_{2}^{-1} \in Z_{\varphi}$. Hence $\psi_{1}^{*} \theta-\theta=\psi_{2}^{*} \tau^{*} \theta-\theta=\psi_{2}^{*} \theta-\theta$ which implies $d f_{\psi_{1}}=d f_{\psi_{2}}$. Thus $f_{\psi_{1}}=f_{\psi_{2}}$ since they both vanish at $x_{0}$.

A theorem of Mañé [1996] (see also [Contreras et al. 1997]) ensures that given two distinct points $x$ and $y$ in $\widetilde{M}$ there exists a magnetic geodesic $\gamma:[0, R] \rightarrow \widetilde{M}$ with energy $k$, which connects $x$ to $y$ and realizes the potential, i.e.,

$$
A_{L+k}(\gamma)=\Phi_{k}(x, y) .
$$

On account of (10)

$$
\Phi_{k}(x, y) \geq \varepsilon R \geq \frac{\varepsilon}{\sqrt{2 k}} d(x, y) .
$$


Let $a:=\inf _{x \in \tilde{M}} \Phi_{k}(x, \varphi x)$. Take a sequence of points $x_{n}$ such that $\Phi_{k}\left(x_{n}, \varphi x_{n}\right)$ converges to $a$. Let $K$ be a compact fundamental domain for the action of $\pi_{1}(M)$ on $\tilde{M}$ and let $\psi_{n} \in \pi_{1}(M)$ be such that $\psi_{n}^{-1} x_{n} \in K$. Let $y_{n}:=\psi_{n}^{-1} x_{n}$. Without loss of generality we can assume that $y_{n}$ converges to some point $y \in K$. Using the triangle inequality for $\Phi_{k}$ we have:

$$
\Phi_{k}\left(\psi_{n} y, \varphi \psi_{n} y\right) \leq \Phi_{k}\left(\psi_{n} y, \psi_{n} y_{n}\right)+\Phi_{k}\left(\psi_{n} y_{n}, \varphi \psi_{n} y_{n}\right)+\Phi_{k}\left(\varphi \psi_{n} y_{n}, \varphi \psi_{n} y\right) .
$$

Using the $\varphi$-invariance of $\Phi_{k}$ and (11) we obtain

$$
\begin{aligned}
& \Phi_{k}\left(\psi_{n} y, \varphi \psi_{n} y\right) \\
& \quad \leq \Phi_{k}\left(y, y_{n}\right)+f_{\psi_{n}}\left(y_{n}\right)-f_{\psi_{n}}(y)+\Phi_{k}\left(y_{n}, y\right)+f_{\psi_{n}}(y)-f_{\psi_{n}}\left(y_{n}\right)+\Phi_{k}\left(x_{n}, \varphi x_{n}\right) \\
& \quad=\Phi_{k}\left(y, y_{n}\right)+\Phi_{k}\left(y_{n}, y\right)+\Phi_{k}\left(x_{n}, \varphi x_{n}\right) .
\end{aligned}
$$

But the expression $\Phi_{k}\left(y, y_{n}\right)+\Phi_{k}\left(y_{n}, y\right)+\Phi_{k}\left(x_{n}, \varphi x_{n}\right)$ is bounded in $n$; hence there exists $C>0$ such that

$$
\Phi_{k}\left(\psi_{n} y, \varphi \psi_{n} y\right)<C
$$

for all $n$. Inequality (12) now implies that there exist only finitely many different elements of the form $\psi_{n}^{-1} \varphi \psi_{n}$. Hence, for infinitely many values of $n, \psi_{n}^{-1} \varphi \psi_{n}$ equals some fixed covering transformation, say $\lambda$. Without loss of generality we shall assume that $\psi_{n}^{-1} \varphi \psi_{n}=\lambda$ for all $n$. Lemma 5.2 tells us that $f_{\psi_{n}}$ is independent of $n$, so we set $f_{0}:=f_{\psi_{n}}$.

Using (11) again we have

$$
\begin{aligned}
\Phi_{k}\left(\psi_{n} y, \varphi \psi_{n} y\right) & =\Phi_{k}(y, \lambda y)+f_{0}(\lambda y)-f_{0}(y), \\
\Phi_{k}\left(x_{n}, \varphi x_{n}\right) & =\Phi_{k}\left(\psi_{n} y_{n}, \varphi \psi_{n} y_{n}\right)=\Phi_{k}\left(y_{n}, \lambda y_{n}\right)+f_{0}\left(\lambda y_{n}\right)-f_{0}\left(y_{n}\right) .
\end{aligned}
$$

Since $\Phi_{k}\left(x_{n}, \varphi x_{n}\right)$ converges to $a$ we conclude that $\Phi_{k}\left(\psi_{n} y, \varphi \psi_{n} y\right)=a$ for all $n$, hence the points $\psi_{n} y$ realize the infimum of the function $x \mapsto \Phi_{k}(x, \varphi x)$.

Let $z$ be one of these points, so that $\Phi_{k}(z, \varphi z)=a$. Consider the minimizing magnetic geodesic $\gamma$ with energy $k$ given by Mañés theorem, connecting $z$ to $\varphi z$ and for which

$$
A_{L+k}(\gamma)=\Phi_{k}(z, \varphi z) .
$$

We claim that $d \varphi(\dot{\gamma}(0))=\dot{\gamma}(R)$. This implies that the projection of $\gamma$ to $M$ gives a closed magnetic geodesic in the free homotopy class determined by $\varphi$. To prove that $d \varphi(\dot{\gamma}(0))=\dot{\gamma}(R)$ we play the same game as in Riemannian geometry. Consider $b>0$ small and note that

$$
\begin{aligned}
\Phi_{k}(\gamma(b), \varphi \gamma(b)) & \leq \Phi_{k}(\gamma(b), \varphi z)+\Phi_{k}(\varphi z, \varphi \gamma(b)) \\
& =\Phi_{k}(\gamma(b), \varphi z)+\Phi_{k}(z, \gamma(b))=\Phi_{k}(z, \varphi z),
\end{aligned}
$$


where in the first equality we used the $\varphi$-invariance of $\Phi_{k}$. Since $x \mapsto \Phi_{k}(x, \varphi x)$ achieves its minimum at $z$, we must have $d \varphi(\dot{\gamma}(0))=\dot{\gamma}(R)$, which concludes the proof of the theorem.

The next lemma will be important for us. Its proof is a fairly standard application of amenability combined with smoothing results of [Fathi and Maderna $\geq 2007$ ].

Lemma 5.3. Let $\Gamma \subset \pi_{1}(M)$ be an amenable subgroup. For any $k>c$, there exists a smooth $\Gamma$-invariant primitive $\vartheta$ such that

$$
\sup _{x \in M} \frac{1}{2}\left|\vartheta_{x}\right|^{2} \leq k
$$

Proof. Take $\widehat{M}=\tilde{M} / \Gamma$. Let $C^{*}(\tilde{M})$ and $C^{*}(\widehat{M})$ be the real cochains (on smooth chains) in $\widetilde{M}$ and $\widehat{M}$ respectively. We can view $C^{*}(\widehat{M})$ as the set of $\Gamma$-invariant cochains in $\widetilde{M}$. Given a manifold $X$, let $\Psi: \Omega^{*}(X) \rightarrow C^{*}(X)$ be the de Rham map:

$$
\Psi(\omega)(\sigma)=\int_{\sigma} \omega .
$$

It induces an isomorphism in real cohomology.

By the definition of Mañé's critical value, given $k>c$, there exists a smooth primitive $\theta$ and $\varepsilon>0$ such that

$$
\sup _{x \in M} \frac{1}{2}\left|\theta_{x}\right|^{2}=\frac{1}{2}\|\theta\|_{\infty}^{2} \leq k-\varepsilon .
$$

Note that

$$
|(\Psi \theta)(\sigma)| \leq\|\theta\|_{\infty} \ell(\sigma),
$$

where $\ell(\sigma)$ is the length of $\sigma$. The group $\Gamma$ acts on $C^{*}(\tilde{M})$ by $\left(\varphi^{*} c\right)(\sigma)=c(\varphi \circ \sigma)$, which gives $\varphi^{*}(\Psi \theta)(\sigma)=\Psi\left(\varphi^{*} \theta\right)(\sigma)$. Since $\Gamma$ acts by isometries, we obtain

$$
\left|\varphi^{*}(\Psi \theta)(\sigma)\right| \leq\|\theta\|_{\infty} \ell(\sigma) .
$$

Thus, for a fixed $\sigma$, the map $\varphi \mapsto \varphi^{*}(\Psi \theta)(\sigma)$ is in $\ell^{\infty}(\Gamma)$.

Since $\Gamma$ is amenable it has a right invariant mean on $\ell^{\infty}(\Gamma)$, that is, there exists a bounded linear functional $m: \ell^{\infty}(\Gamma) \rightarrow \mathbb{R}$ such that

(i) $m(a)=a$ if $a$ is a constant function;

(ii) $m\left(a_{1}\right) \geq m\left(a_{2}\right)$ if $a_{1}(\varphi) \geq a_{2}(\varphi)$ for all $\varphi \in \Gamma$;

(iii) $m\left(\varphi_{*} a\right)=m(a)$, where $\varphi_{*} a(\psi):=a(\psi \varphi)$.

Now define

$$
A_{\theta}(\sigma):=m\left(\varphi \mapsto \varphi^{*}(\Psi \theta)(\sigma)\right) .
$$

Clearly $A_{\theta}$ lies in $C^{*}(\tilde{M})$ and is $\Gamma$-invariant. It also satisfies

$$
\left|A_{\theta}(\sigma)\right| \leq\|\theta\|_{\infty} \ell(\sigma) .
$$


Let $\delta$ be the boundary operator on cochains. We have

$$
\left(\delta A_{\theta}\right)(\sigma)=A_{\theta}(\partial \sigma)=m\left(\varphi \mapsto \varphi^{*}(\Psi \theta)(\partial \sigma)\right) .
$$

But the value of

$$
\varphi^{*}(\Psi \theta)(\partial \sigma)=\int_{\partial \sigma} \varphi^{*} \theta=\int_{\sigma} d\left(\varphi^{*} \theta\right)=\int_{\sigma} \varphi^{*} \Pi^{*} \Omega=\int_{\sigma} \Pi^{*} \Omega
$$

is independent of $\varphi$. Thus

which implies

$$
\left(\delta A_{\theta}\right)(\sigma)=\int_{\sigma} \Pi^{*} \Omega=\Psi\left(\Pi^{*} \Omega\right)(\sigma),
$$

$$
\delta A_{\theta}=\Psi\left(\Pi^{*} \Omega\right) .
$$

Since both $A_{\theta}$ and $\Pi^{*} \Omega$ descend to $\widehat{M}$ to give $\widehat{A}_{\theta}$ and $\widehat{\Omega}$, de Rham's theorem tells us that $\widehat{\Omega}$ is exact. Hence there exists a smooth 1 -form $\alpha$ in $\widehat{M}$ such that $\widehat{\Omega}=d \alpha$. Now $\widehat{A}_{\theta}-\Psi \alpha$ is a 1-cocycle in $\widehat{M}$ and so there is a smooth closed 1-form $\omega$ in $\widehat{M}$ such that $\Psi(\omega+\alpha)$ and $\widehat{A}_{\theta}$ are cohomologous. Thus for any 1-cycle $\sigma$ we have

$$
\widehat{A}_{\theta}(\sigma)=\int_{\sigma} \alpha+\omega,
$$

which in turn implies by (14) that, for any smooth closed curve $\gamma$ in $\widehat{M}$,

$$
\left|\int_{\gamma} \alpha+\omega\right| \leq\|\theta\|_{\infty} \ell(\gamma) \text {. }
$$

On account of (13) this implies that the action of the Lagrangian

$$
\frac{1}{2}|v|_{x}^{2}+\alpha_{x}(v)+\omega_{x}(v)+k-\varepsilon
$$

on any closed curve $\gamma:[0, T] \rightarrow \widehat{M}$ is at least

$$
\frac{\ell(\gamma)^{2}}{2 T}-\|\theta\|_{\infty} \ell(\gamma)+\frac{\|\theta\|_{\infty}^{2} T}{2}=\left(\frac{\ell(\gamma)}{\sqrt{2 T}}-\frac{\|\theta\|_{\infty} \sqrt{T}}{\sqrt{2}}\right)^{2} \geq 0 .
$$

This is enough to ensure the existence of a locally Lipschitz function $u: \widehat{M} \rightarrow \mathbb{R}$ such that, for almost every $x \in \widehat{M}$

$$
\frac{1}{2}\left|\alpha_{x}+\omega_{x}+d_{x} u\right|^{2} \leq k-\varepsilon .
$$

(See [Burns and Paternain 2002] for a proof.) But now the smoothing results in [Fathi and Maderna $\geq 2007$, Section 6] imply that there exists a smooth function $u_{\varepsilon}: \widehat{M} \rightarrow \mathbb{R}$ such that

$$
\frac{1}{2}\left|\alpha_{x}+\omega_{x}+d_{x} u_{\varepsilon}\right|^{2} \leq k
$$

for all $x \in \widehat{M}$. If we let $\vartheta$ be the lift of $\alpha+\omega+d u_{\varepsilon}$ to $\tilde{M}$, we obtain a smooth 1 -form possessing all the required properties. 
Corollary 5.4. Suppose that $\pi_{1}(M)$ is amenable and $\Omega$ is not exact. Then $c(g, \Omega)$ is infinite.

Proof. If $c(g, \Omega)$ is finite, $\widetilde{\Omega}$ admits a bounded primitive and by the previous lemma, $\widetilde{\Omega}$ admits a $\pi_{1}(M)$-invariant primitive $\theta$. The form $\theta$ descends to $M$, showing that $\Omega$ is exact.

Theorem 5.5. Take $k>c$ and let $\varphi \in \pi_{1}(M)$ be a nontrivial element with amenable centralizer. Then the nontrivial free homotopy class determined by $\varphi$ contains a closed magnetic geodesic with energy $k$.

Proof. This follows right away from Theorem 5.1 and Lemma 5.3.

Remark 5.6. If $\pi_{1}(M)$ is the fundamental group of a closed manifold of negative curvature and $\varphi$ is nontrivial, Preissman's theorem implies that $Z_{\varphi}$ coincides with the infinite cyclic group generated by $\varphi$, which is of course amenable. Thus we can apply Theorem 5.5 to any nontrivial free homotopy class.

In the next section we will apply the theorem to a closed surface of genus $\geq 2$.

Proof of Theorem C. Theorem C is an immediately consequence of Theorem 5.5 and the following result, which was proved by G. Contreras [2003] in the exact case and extended by O. Osuna [2005] to the weakly exact case, as part of his Ph.D. work.

Theorem 5.7. Suppose the lift of $\Omega$ to $\widetilde{M}$ has a bounded primitive. Then, for almost every $k$ in the interval $(0, c)$ there exists a closed contractible magnetic geodesic with energy $k$.

This is proved by showing that an appropriate action functional (which depends on the energy level) on the space of contractible loops exhibits a mountain pass geometry. Standard Morse theory then guarantees that critical points exist whenever the Palais-Smale condition holds. It has been known for some time that the Palais-Smale condition can only fail in the time direction. An argument originally due to M. Struwe can now be applied to the mountain pass geometry to overcome this difficulty for almost every energy level.

\section{Proof of Theorem B}

In this section we return to the case in which $M$ is a closed surface of genus $\geq 2$. The following lemma has independent interest.

Lemma 6.1. Suppose the magnetic flow of $(g, \Omega)$ is Mañé-critical. If there exists a nontrivial free homotopy class without closed magnetic geodesics, then there exists an invariant Borel probability measure $v$ with $\mathfrak{a}(v)=\mathscr{S}(v)=0$. Equivalently, the magnetic flow is not of contact type. 
Proof. Let $\sigma$ be a nontrivial free homotopy class without closed magnetic geodesics and suppose that $\sigma$ is generated by the covering transformation $\varphi$. As in the proof of Theorem 5.5 we consider a $\varphi$-invariant action potential $\Phi_{k} \geq 0$ for all $k>c=\frac{1}{2}$. Now take a decreasing sequence $k_{n}$ approaching $\frac{1}{2}$ as $n \rightarrow \infty$. Theorem 5.5 gives points $x_{n}$ and orbits $\gamma_{n}:\left[0, T_{n}\right] \rightarrow \widetilde{M}$ with energy $k_{n}$ connecting $x_{n}$ and $\varphi x_{n}$. The orbits $\gamma_{n}$ project to $M$ as closed orbits in the class $\sigma$ and

$$
0 \leq A_{L_{n}+k_{n}}\left(\gamma_{n}\right)=\Phi_{k_{n}}\left(x_{n}, \varphi x_{n}\right) .
$$

Moreover, $x_{n}$ is a minimum of $x \mapsto \Phi_{k_{n}}(x, \varphi x)$. Hence, if $y$ is any point in $\tilde{M}$,

$$
\Phi_{k_{n}}\left(x_{n}, \varphi x_{n}\right) \leq \Phi_{k_{n}}(y, \varphi y) \leq C
$$

for some constant $C>0$, since $L_{n}(x, v)+k_{n} \leq \frac{1}{2}+k_{n}+\sqrt{2 k_{n}}$ for all $(x, v) \in T M$ with $|v|_{x} \leq 1$. Thus

$$
0 \leq A_{L_{n}+k_{n}}\left(\gamma_{n}\right) \leq C .
$$

We now observe that $\inf _{n} T_{n}>0$, otherwise we would get curves in the class $\sigma$ with arbitrarily short lengths, which is impossible. If $\sup _{n} T_{n}<\infty$, by passing to a subsequence if necessary, we can assume that $T_{n} \rightarrow T_{0}$ and that the projection of $\left(\gamma_{n}(0), \dot{\gamma}_{n}(0)\right)$ to $T M$ converges to some point $(p, v) \in S M$. The orbit of $(p, v)$ gives rise to a closed magnetic geodesic with period $T_{0}$ in the homotopy class $\sigma$. Since we are assuming that $\sigma$ has no such orbits we must have $\sup _{n} T_{n}=\infty$. Without loss of generality we shall assume from now on that $T_{n} \rightarrow \infty$.

We indicate with a tilde the lift of objects on $M$ (or $S M$ ) to $\widetilde{M}$ (or $S \tilde{M}$ ). Note that

$$
\left(L_{n}+k_{n}\right)\left(\gamma_{n}, \dot{\gamma}_{n}\right)=2 k_{n}-\theta_{n}\left(\dot{\gamma}_{n}\right)=\left(\tilde{\alpha}-\tilde{\pi}^{*} \theta_{n}\right)(\tilde{X})\left(\gamma_{n}, \dot{\gamma}_{n}\right) .
$$

Let $\Theta$ be a primitive of $\omega$ in a neighborhood of $S M$. Since

$$
d\left(\tilde{\alpha}-\tilde{\pi}^{*} \theta_{n}\right)=-\tilde{\omega}=-d \widetilde{\Theta},
$$

there exists a smooth closed 1 -form $\rho_{n}$ defined in a neighborhood of $S \tilde{M}$ for which

$$
\tilde{\alpha}-\tilde{\pi}^{*} \theta_{n}=-\widetilde{\Theta}+\rho_{n}
$$

Combining (16) and (17) we obtain

$$
\left(L_{n}+k_{n}\right)\left(\gamma_{n}, \dot{\gamma}_{n}\right)=-\widetilde{\Theta}(\tilde{X})\left(\gamma_{n}, \dot{\gamma}_{n}\right)+\rho_{n}(\tilde{X})\left(\gamma_{n}, \dot{\gamma}_{n}\right) .
$$

Let $v_{n}$ be the Borel probability measure on $T M$ given by

$$
\int f d v_{n}:=\frac{1}{T_{n}} \int_{0}^{T_{n}} f\left(\Pi\left(\gamma_{n}(t)\right), d \Pi\left(\dot{\gamma}_{n}(t)\right)\right) d t .
$$


Without loss of generality we can assume that $v_{n}$ converges weakly to an invariant measure $v$. Since $k_{n} \rightarrow \frac{1}{2}$, the measure $v$ has support in $S M$. We check that $\mathfrak{a}(v)=\mathscr{Y}(v)=0$. Using (15) and (18) we see that

$$
0=\lim \frac{1}{T_{n}} A_{L_{n}+k_{n}}\left(\gamma_{n}\right)=-\int \Theta(X) d v+\lim \frac{1}{T_{n}} \int_{\left(\gamma_{n}, \dot{\gamma_{n}}\right)} \rho_{n},
$$

and therefore to show that $\mathfrak{a}(v)=0$ it suffices to check that

$$
\lim \frac{1}{T_{n}} \int_{\left(\gamma_{n}, \dot{\gamma}_{n}\right)} \rho_{n}=0 .
$$

Equality (17) implies that $\rho_{n}$ is $\varphi$-invariant and its norm is bounded by a constant independent of $n$, say $A$. Let $\widehat{M}$ be the manifold obtained by taking the quotient of $\widetilde{M}$ by the action of the cyclic group generated by $\varphi$. The curves $\gamma_{n}$ project to simple closed curves in $\widehat{M}$ that are all homotopic, so the curves $\left(\gamma_{n}, \dot{\gamma}_{n}\right)$ in $T \tilde{M}$ project to closed curves $\Gamma_{n}$ in a neighborhood of $S \widehat{M}$ whose homology class $\left[\Gamma_{n}\right]$ is independent of $n$. The form $\rho_{n}$ descends to a closed 1-form $\hat{\rho}_{n}$ defined in a neighborhood of $S \widehat{M}$. Observe that

$$
\int_{\left(\gamma_{n}, \dot{\gamma}_{n}\right)} \rho_{n}=\int_{\Gamma_{n}} \hat{\rho}_{n}=\left\langle\left[\hat{\rho}_{n}\right],\left[\Gamma_{n}\right]\right\rangle .
$$

Since $\left[\Gamma_{n}\right]$ is independent of $n$ and $\hat{\rho}_{n}$ is bounded by $A$ we have

$$
\lim \frac{1}{T_{n}}\left\langle\left[\hat{\rho}_{n}\right],\left[\Gamma_{n}\right]\right\rangle=0,
$$

as desired.

Next we prove that $\mathscr{S}(\nu)=0$. Let $\Upsilon$ be any closed 1-form on $S M$. Since $\pi^{*}: H^{1}(M, \mathbb{R}) \rightarrow H^{1}(S M, \mathbb{R})$ is an isomorphism, there exists a closed 1-form $\delta$ on $M$ and a smooth function $G$ on $S M$ such that $\Upsilon=\pi^{*} \delta+d G$. Thus

$$
\int \Upsilon(X) d v=\int \pi^{*} \delta(X) d v
$$

and to prove that $\mathscr{Y}(v)=0$ it suffices to show that

$$
\int \pi^{*} \delta(X) d v=0
$$

But

$$
\int \pi^{*} \delta(X) d v=\lim \frac{1}{T_{n}} \int_{\Pi \circ \gamma_{n}} \delta=\lim \frac{1}{T_{n}}\left\langle[\delta],\left[\Pi \circ \gamma_{n}\right]\right\rangle .
$$

The curves $\Pi \circ \gamma_{n}$ are all in the same free homotopy class $\sigma$, hence the homology class $\left[\Pi \circ \gamma_{n}\right]$ is independent of $n$ which gives $\mathscr{Y}(v)=0$, as desired. 
To complete the proof of the lemma, recall that $S M$ is of contact type if and only if for all invariant Borel probability measures $\mu$ with zero asymptotic cycle, $\mathfrak{a}(\mu) \neq 0$; see [Contreras et al. 2004, Proposition 2.4].

Proof of Theorem B. First, we always have semistatic curves starting at any point in $\widetilde{M}$ [Contreras et al. 1998; Contreras 2001; Fathi and Maderna $\geq 2007$ ]. These are magnetic geodesics $\gamma:[0, \infty) \rightarrow \widetilde{M}$ such that

$$
A_{L+1 / 2}\left(\left.\gamma\right|_{[s, t]}\right)=\Phi_{1 / 2}(\gamma(s), \gamma(t))
$$

for $0 \leq s<t<\infty$ where as before

$$
\Phi_{1 / 2}(x, y)=\inf _{T>0} \Phi_{1 / 2}(x, y ; T) .
$$

A semistatic curve must be free of conjugate points in $[0, \infty)$, by [Contreras and Iturriaga 1999, Corollary 4.2]; hence the $\omega$-limit set of the projection of $\gamma$ to $M$ must also be free of conjugate points. Since we are assuming that the magnetic flow is uniquely ergodic, this implies that all $S M$ is free of conjugate points. On account of Lemma 6.1 and unique ergodicity, $\mathfrak{a}\left(\mu_{\ell}\right)=0$ and the theorem follows from the Proposition.

Remark 6.2. To obtain the conclusion of Theorem B it is not really necessary to assume unique ergodicity, merely that there is a unique invariant probability measure with zero asymptotic cycle. The measure $v$ obtained in Lemma 6.1 will have zero asymptotic Maslov index because it is the limit of measures $v_{n}$ with zero asymptotic Maslov index (the asymptotic Maslov index is continuous in the weak* topology [Contreras et al. 2003]). The measures $v_{n}$ have zero asymptotic Maslov index because they are supported on the minimizers $\Pi \circ \gamma_{n}$ and we are dealing with surfaces.

Remark 6.3. We can rephrase Lemma 6.1 by saying that if a Mañé-critical magnetic flow is of contact type, every nontrivial free homotopy class contains a closed magnetic geodesic. Since there are nontrivial free homotopy classes with the property that any closed curve in them is homologous to zero, we obtain, in particular, closed magnetic geodesics homologous to zero. Recall that the Weinstein conjecture says that every Reeb vector field on a closed 3-manifold admits a closed orbit. The strong Weinstein conjecture asserts that in fact one can find finitely many closed orbits which form a cycle homologous to zero. There has been much progress recently regarding this conjecture; see [Abbas et al. 2005]. However, the work of J. Etnyre [2004] implies that magnetic flows, with $\Omega$ symplectic, are excluded from all known cases in which the conjecture holds. 


\section{Acknowledgments}

I thank Leonardo Macarini for several useful comments and discussions about Theorem C. He suggested the possibility of using Contreras' recent results to prove statements like Theorem C. I also thank Viktor Ginzburg and Felix Schlenk for several discussions regarding the question of existence of closed magnetic geodesics. Finally I thank the referee for various comments and suggestions that helped improve the paper.

\section{References}

[Abbas et al. 2005] C. Abbas, K. Cieliebak, and H. Hofer, "The Weinstein conjecture for planar contact structures in dimension three", Comment. Math. Helv. 80:4 (2005), 771-793. MR 2006g:53135 Zbl 02242662

[Arnol'd 1961] V. I. Arnol'd, "Some remarks on flows of line elements and frames", Dokl. Akad. Nauk SSSR 138 (1961), 255-257. In Russian; translated in Sov. Math. Dokl. 2 (1961), 562-564. MR 28 \#1555 Zbl 0124.14601

[Bialy 2000] M. L. Bialy, "Rigidity for periodic magnetic fields", Ergodic Theory Dynam. Systems 20:6 (2000), 1619-1626. MR 2002e:37080 Zbl 1003.37035

[Burger 1990] M. Burger, "Horocycle flow on geometrically finite surfaces", Duke Math. J. 61:3 (1990), 779-803. MR 91k:58102 Zbl 0723.58041

[Burns and Paternain 2002] K. Burns and G. P. Paternain, "Anosov magnetic flows, critical values and topological entropy”, Nonlinearity 15:2 (2002), 281-314. MR 2004d:37076 Zbl 01753660

[Contreras 2001] G. Contreras, "Action potential and weak KAM solutions", Calc. Var. Partial Differential Equations 13:4 (2001), 427-458. MR 2002j:37092 Zbl 0993.37030

[Contreras 2003] G. Contreras, "The Palais-Smale condition on contact type energy levels for convex Lagrangian systems", preprint, 2003. To appear in Calc. Var. Partial Differential Equations. math.DS/0304238

[Contreras and Iturriaga 1999] G. Contreras and R. Iturriaga, "Convex Hamiltonians without conjugate points”, Ergodic Theory Dynam. Systems 19:4 (1999), 901-952. MR 2000h:37102 Zbl 1044. 37046

[Contreras et al. 1997] G. Contreras, J. Delgado, and R. Iturriaga, "Lagrangian flows: the dynamics of globally minimizing orbits. II”, Bol. Soc. Brasil. Mat. (N.S.) 28:2 (1997), 155-196. MR 98i:58093 Zbl 0892.58065

[Contreras et al. 1998] G. Contreras, R. Iturriaga, G. P. Paternain, and M. Paternain, "Lagrangian graphs, minimizing measures and Mañé's critical values”, Geom. Funct. Anal. 8:5 (1998), 788-809. MR 99f:58075 Zbl 0920.58015

[Contreras et al. 2003] G. Contreras, J.-M. Gambaudo, R. Iturriaga, and G. P. Paternain, "The asymptotic Maslov index and its applications", Ergodic Theory Dynam. Systems 23:5 (2003), 1415-1443. MR 2004k:37104 Zbl 1052.53055

[Contreras et al. 2004] G. Contreras, L. Macarini, and G. P. Paternain, "Periodic orbits for exact magnetic flows on surfaces", Int. Math. Res. Not. 8 (2004), 361-387. MR 2005a:37103 Zbl 02207647

[Croke and Kleiner 1994] C. B. Croke and B. Kleiner, "Conjugacy and rigidity for manifolds with a parallel vector field”, J. Differential Geom. 39:3 (1994), 659-680. MR 95a:53064 Zbl 0807.53035 [Etnyre 2004] J. B. Etnyre, "Planar open book decompositions and contact structures", Int. Math. Res. Not. 79 (2004), 4255-4267. MR MR2126827 (2005k:57049) Zbl 1069.57016 
[Fathi $\geq 2007$ ] A. Fathi, The weak KAM theorem and Lagrangian dynamics, Cambridge University Press, Cambridge. To appear.

[Fathi and Maderna $\geq 2007$ ] A. Fathi and E. Maderna, "Weak KAM theorem on non compact manifolds", Nonlinear Differ. Equ. Appl..

[Flaminio and Forni 2003] L. Flaminio and G. Forni, "Invariant distributions and time averages for horocycle flows", Duke Math. J. 119:3 (2003), 465-526. MR 2004g:37039 Zbl 1044.37017

[Furstenberg 1973] H. Furstenberg, "The unique ergodicity of the horocycle flow", pp. 95-115 in Recent advances in topological dynamics: in honor of Gustav Arnold Hedlund (New Haven, 1972), edited by A. Beck, Lecture Notes in Math. 318, Springer, Berlin, 1973. MR 52 \#14149 Zbl 0256.58009

[Ginzburg 1995] V. L. Ginzburg, "An embedding $S^{2 n-1} \rightarrow \mathbf{R}^{2 n}, 2 n-1 \geq 7$, whose Hamiltonian flow has no periodic trajectories", Internat. Math. Res. Notices 2 (1995), 83-97. MR 96a:58087 Zbl 0835.58029

[Ginzburg 1996] V. L. Ginzburg, "On the existence and non-existence of closed trajectories for some Hamiltonian flows", Math. Z. 223:3 (1996), 397-409. MR 97i:58144 Zbl 0862.58020

[Ginzburg 1997] V. L. Ginzburg, "A smooth counterexample to the Hamiltonian Seifert conjecture in $\mathbf{R}^{6}$ ”, Internat. Math. Res. Notices 13 (1997), 641-650. MR 98i:58087 Zbl 0913.58022

[Ginzburg and Gürel 2003] V. L. Ginzburg and B. Z. Gürel, "A $C^{2}$-smooth counterexample to the Hamiltonian Seifert conjecture in $\mathbb{R}^{4}$ ", Ann. of Math. (2) 158:3 (2003), 953-976. MR 2005e:37133 Zbl pre02068687

[Ginzburg and Kerman 1999] V. L. Ginzburg and E. Kerman, "Periodic orbits in magnetic fields in dimensions greater than two", pp. 113-121 in Geometry and topology in dynamics (Winston-Salem, NC, 1998 and San Antonio, TX, 1999), edited by M. Barge and K. Kuperberg, Contemp. Math. 246, Amer. Math. Soc., Providence, RI, 1999. MR 2000k:37087 Zbl 0948.37045

[Gouda 1998] N. Gouda, “The theorem of E. Hopf under uniform magnetic fields", J. Math. Soc. Japan 50:3 (1998), 767-779. MR 99f:53041 Zbl 0914.53023

[Green 1958] L. W. Green, “A theorem of E. Hopf”, Michigan Math. J. 5 (1958), 31-34. MR 20 \#4300 Zbl 0134.39601

[Gromov 1991] M. Gromov, "Kähler hyperbolicity and $L_{2}$-Hodge theory", J. Differential Geom. 33:1 (1991), 263-292. MR 92a:58133 Zbl 0719.53042

[Hopf 1948] E. Hopf, "Closed surfaces without conjugate points", Proc. Nat. Acad. Sci. USA 34 (1948), 47-51. MR 9,378d Zbl 0030.07901

[Macarini 2003] L. Macarini, "Hofer-Zehnder semicapacity of cotangent bundles and symplectic submanifolds", preprint A/2003/242, IMPA, Rio de Janeiro, 2003. math.SG/0303230

[Mañé 1996] R. Mañé, "Lagrangian flows: the dynamics of globally minimizing orbits”, pp. 120131 in International Conference on Dynamical Systems: a tribute to Ricardo Mañé, edited by F. Ledrappier et al., Pitman Research Notes in Math. 362, Longman, Harlow, 1996. Reprinted in Bol. Soc. Brasil. Mat. (N.S.) 28:2 (1997), 141-153. MR 98i:58092 Zbl 0870.58026

[Marcus 1978] B. Marcus, “The horocycle flow is mixing of all degrees", Invent. Math. 46:3 (1978), 201-209. MR 58 \#7731 Zbl 0395.28012

[Marcus 1983] B. Marcus, "Topological conjugacy of horocycle flows", Amer. J. Math. 105:3 (1983), 623-632. MR 85b:58103 Zbl 0533.58029

[Osuna 2005] O. Osuna, "Periodic orbits of weakly exact magnetic flows", preprint, 2005.

[Paternain and Paternain 1996] G. P. Paternain and M. Paternain, "Anosov geodesic flows and twisted symplectic structures", pp. 132-145 in International Conference on Dynamical Systems: 
a tribute to Ricardo Mañé (Montevideo, 1995), edited by F. Ledrappier et al., Pitman Res. Notes Math. Ser. 362, Longman, Harlow, 1996. MR 98h:58145 Zbl 0868.58062

[Pier 1984] J.-P. Pier, Amenable locally compact groups, Pure and Applied Mathematics, Wiley, New York, 1984. MR 86a:43001 Zbl 0621.43001

[Ratner 1982] M. Ratner, "Rigidity of horocycle flows", Ann. of Math. (2) 115:3 (1982), 597-614. MR 84e:58062 Zbl 0506.58030

[Ratner 1983] M. Ratner, "Horocycle flows, joinings and rigidity of products", Ann. of Math. (2) 118:2 (1983), 277-313. MR 85k:58063 Zbl 0556.28020

[Schlenk 2006] F. Schlenk, “Applications of Hofer's geometry to Hamiltonian dynamics", Comm. Math. Helv. 81 (2006), 105-121.

Received October 8, 2004. Revised May 23, 2005.

GABRIEl P. PATERnAin

Department of Pure Mathematics and Mathematical Statistics

UNIVERSITY OF CAMBRIDGE

CAMBRIDGE CB3 0WB

ENGLAND

g.p.paternain@dpmms.cam.ac.uk 